\title{
A consideration of modelling techniques that can be used to evaluate manufacturing strategies
}

\section{T. S. Baines, D. K. Harrison, J. M. Kay and D. J. Hamblin}

\section{Introduction}

The management activity of striving to reach a congruence between manufacturing capabilities and the demands of market and financial environments is often referred to as manufacturing strategy formulation and implementation. The manufacturing capabilities establish the competitiveness of a company's products and can be measured in terms of such variables as service, quality, and cost. These capabilities are determined by the overall mix of processes, technology, human resources, systems, etc., of which a production facility consists. The chosen mix can be referred to as the company's manufacturing strategy.

Methodologies exist to help practising engineers to formulate manufacturing strategies, see for example [1]. These processes usually follow a sequence of activities that secure recognition of a company's existing manufacturing capabilities, structure an expression of the associated financial and market environments, and stimulate the choice of processes, technology, etc., to overcome any deficits that may exist. Inherent to such processes is some form of evaluation where an attempt is made to understand the effects of a proposed manufacturing strategy on the performance of the host production facility. Evaluation is usually carried out through some combination of judgement, bargaining and analysis. Analysis may include building a model that emulates the behaviour of a manufacturing system, which can then be used to gain insight into and make predictions about that system. Such a model can then be used to support judgement and bargaining between industrialists in the evaluation process.

There is no universally accepted best modelling technique for the task of manufacturing strategy evaluation. Rather, there are a variety of model-building approaches from which a manager must choose. The literature provides a rich source of information and advice across a broad spectrum of model applications, but unfortunately this also contains some unsupported claims and contradictions. This can be a handicap to the practising manager who is considering using a modelling technique in the analysis of strategic changes to a manufacturing system. Therefore, the work described in this paper has set out to remedy this situation through conducting a structured and comparative assessment of modelling approaches that can be used for evaluating manufacturing strategies. This assessment has been carried out across a broad range of modelling techniques that are popular in the literature. This work has enabled the comparable capabilities and characteristics of these modelling techniques to be determined and presented in this paper.

The assessment programme has focused exclusively on the performance of a range of modelling techniques in the task of evaluating manufacturing strategies. To achieve this, seven modelling techniques have been chosen as being representative of those popular in the literature. These modelling techniques are IDEFo, enterprise modelling, discrete event simulation, system dynamics, queuing theory, business planning and activity based costing. An industrially based experiment has then been conducted where each modelling technique has been used to analyse a number of strategic scenarios for a real manufacturing system. This has then enabled the characteristics of each technique to be summarised against capabilities that are believed to be important in a modelling technique for manufacturing strategy formulation. On this basis, this paper is structured to provide a description of the strategy evaluation task, followed by an overview of the experimental study, and then to present the findings of this work.

\section{Background to Manufacturing Strategy Evaluation Using Modelling}

A manufacturing strategy can be explicitly formed by a number of methods. An approach that has been seen to be particularly successful is strategy formulation by a formal planning process [2]. Usually, such a process is a sequence of planning activities that help managers to clearly describe a company's existing manufacturing capabilities, along 
with the associated financial and market environments. The process then guides managers to construct a strategic plan for the company's manufacturing function.

Evaluation is a principal stage in strategy formulation [3]. Such evaluation can be made through judgement of executive personnel, refined through bargaining between a number of executives, and supported by analytical methods [4]. One such analytical method is a model that emulates the behaviour of a system, and which can be used to gain insight into, and make predictions about, that system [5]. Hence, if a suitable model of a manufacturing system could be created, a number of modifications could be made to the model to reflect the strategy under consideration, and the ensuing model behaviour could give a prediction of future manufacturing capabilities. Such a model could then be used to support judgement and bargaining amongst executives in the strategy evaluation process. Modelling is often used in detailed design of manufacturing systems [5-7]. However, manufacturing strategy formulation is different from detailed manufacturing system design. Indeed, there are very few instances of research having explicitly investigated how modelling can be used as an aid in manufacturing strategy evaluation [t,8]. One reason for this is that the requirements of modelling in this situation have not previously been explicitly defined.

To understand the requirements of modelling, it is necessary to explore how a model can be used in the evaluation of a manufacturing system. First, a model can be created of the existing manufacturing system. Such a model should be capable of modification to reflect the implementation of a manufacturing strategy. The resulting model behaviour could then be assessed against the performance measures that are appropriate to manufacturing strategy formulation. In this way a number of requirements of a model become apparent. First, a modelling technique should be flexible enough to evaluate a wide variety of changes to processes, technology, human resources, systems, etc., that are associated with a manufacturing strategy. These can be considered as structural and infrastructural changes, and a detailed description of these is given by Platts [2]. Likewise, a modelling technique should enable assessment in terms of strategic market, finance and manufacturing-based performance measures. Again, Platts [2] describes the range of performance measures usually associated with manufacturing strategy. Finally, assessment of the dynamic behaviour of a manufacturing system is also important, as is serviceability in terms of application cost, application time, accuracy, and credibility. On this basis, four factors are required of a modelling technique for it to be suitable for manufacturing strategy evaluation. These categories are:

Assessment of structural and infrastructural changes to a manufacturing system.

Indication of performance in terms of manufacturing objectives. Assessment of system transition.

Serviceability.

\section{The Experiment Aim and Programme}

Practising managers need to know the comparable capabilities of modelling techniques. There is, however, a lack of literature that provides a broad review across the popular yet diverse forms of modelling techniques available to managers. Similarly, empirically based research is rare, as is explicit consideration of the strategy evaluation task. Therefore, the aim of the experimental work reported here has been to accurately determine the comparable capabilities of a wide range of popular modelling techniques when used for the task of analysing manufacturing strategies.

The experimental programme was designed to reveal the performance of a modelling technique against the factors given above. Each factor was taken in turn, and a suitable experiment was determined which would reveal the relevant capabilities of a modelling technique. Once experiments had been established for each factor, these were combined to form the experiment programme. In this way, an experiment programme was designed that consisted of two distinct stages. Both of these stages required modelling to be based on an actual manufacturing system in order to ensure realistic results. The first stage of experiments exposed the functionality of modelling techniques, for example, the performance measurement information supported. This experiment consisted of using each modelling technique to build a model of the complete manufacturing system at the collaborating company (see Section 5 for details about this company). The objective in each case was to build a model that captured the behaviour of the real 
system. Each model was built in three logical stages. Machines were first incorporated, then manpower, and finally material-handling elements added. At each stage, the behaviour of the model was contrasted against that of the actual manufacturing system. This allowed the relationships between model build time and model accuracy to be assessed. Numerous controls were incorporated into the experiments. The data requirements of each modelling technique were defined and appropriate data gathered before model building commenced, and similarly the prior experience of the model builder was accounted for.

The second stage of experiments exposed the flexibility of each modelling technique. This was assessed by attempting to modify each of the models built during the first stage to include a number of strategic manufacturing scenarios. The objective in this case was to construct a valid model of each scenario. These scenarios represented changes to the collaborating company's facilities, capacity, span of process, process and technology, human resources, quality systems, control systems, suppliers, and new product introduction. The actual scenarios applied are shown in Table 1.

Table 1. Summary of the manufacturing scenarios evaluated.

\begin{tabular}{|c|c|}
\hline Decision represented & Overview of manufacturing scenario evaluated \\
\hline Facilities & $\begin{array}{l}\text { Storage of components on assembly line. Removal of storage areas, stocking policy } \\
\text { changed. }\end{array}$ \\
\hline Capacity & $\begin{array}{l}\text { A linear increase in sales volume over } 2 \text { years, so that after this period overall production } \\
\text { has increased by } 40 \% \text {. }\end{array}$ \\
\hline Span of process & $\begin{array}{l}\text { Subcontracting some subassembly operations so that components arrive ready for } \\
\text { assembly onto Product A. }\end{array}$ \\
\hline Processes & $\begin{array}{l}\text { Integrating subassembly operations into main assembly. Setting up subassembly cells } \\
\text { which are spurs off the main assembly line. }\end{array}$ \\
\hline Human resources & Double day shift in assembly and test areas. \\
\hline Quality & $100 \%$ inspection of some products at deliver, to ensure acceptable quality. \\
\hline Control policy & Shop-floor scheduling programme for Product A manufacture. \\
\hline & All Product A supplied "right first time". \\
\hline New products & Product mix changing over 1 year so that Product $\mathrm{C}$ accounts for $40 \%$ of volume. \\
\hline \multicolumn{2}{|l|}{ Note: } \\
\hline 'talier & nufacturing scenarios and their roles in experimentation is given in Baines (8). \\
\hline 2. The ch & arios represent actions actually being considered by the collaborating company. \\
\hline 3. Explicit $r$ & Icts at the collaborating company has been avoided. \\
\hline
\end{tabular}

\section{The Modelling Techniques and Tools Used}

Previous research has sought to identify the wide variety of modelling techniques that are potentially suitable for manufacturing strategy evaluation. This has been achieved by first forming a comprehensive taxonomy of models. Formation of this taxonomy is described in Baines et at. [9], and is summarised in Table 2. The models described in this taxonomy can be principally classed as either physical or symbolic. Baines et al. [8] have argued that only symbolic models are likely to provide the necessary capabilities for analysing manufacturing strategies. In general, this is because construction of physical models is expensive in terms of time and resources. The range of models that can be termed "symbolic" fit into three subclasses. Each subclass can be associated with a number of modelling techniques. For example, system dynamics and discrete event simulation are both forms of simulation modelling techniques. Therefore, the literature has been considered, and popular modelling forms have been chosen to represent each subclass of models, in this way, schematic models are represented in this work by IDEFo and enterprise modelling (EM). Simulation modelling is represented by discrete event simulation (DES) and system dynamics (SD). Similarly, mathematical models are represented by queuing theory (QT), business planning (BP) and activity based costing $(A B C)$. Each modelling technique can be applied in practice using a computer- based modelling tool. The tools chosen for this study included Design/IDEFO, WITNESS, STELLA and MANUPLAN II. Information about these tools is given in [8]. 


\begin{tabular}{lll}
\hline Main class & Subclass & Definition \\
\hline Physical & Replication & $\begin{array}{l}\text { A spatial transform of an original physical object in which the } \\
\text { dimensionality and functionality of the modelling is retained in the replica. } \\
\text { A physical model in which one or more of the dimensions of the physical } \\
\text { object are missing or modified. }\end{array}$ \\
& Quasi-replica & $\begin{array}{l}\text { A model which bears no direct resemblance to the modelled phenomena. } \\
\text { A graphical representation of a system using symbols. }\end{array}$ \\
Symbolic & Schematic & $\begin{array}{l}\text { A model of the behaviour of a system as a whole by defining in detail how } \\
\text { various components interact with each other. } \\
\text { Explicit analytical formulae describing known relationships. }\end{array}$ \\
& Mathematical &
\end{tabular}

\section{The Industrial Test-Bed}

The company chosen for this study was Varsity Perkins Ltd, a British manufacturer of large diesel engines for the marine and electricity generation markets. This is a relatively large company and typical of a multiproduct batch manufacturing environment. In this study, only the Stafford site of this company was considered. This site carries out a wide range of manufacturing activities from initial design through to manufacture and final testing of engines. Many operations aa'e carried out "in-house", although the exception to this is the production of the raw material castings, which are currently contracted out.

A number of company personnel collaborated in this study, for example the manufacturing operations manager, the financial manager, and the manufacturing engineering manager. These personnel were used to assist data collection and validation of each of the models constructed.

\section{Results of Experimentation}

This section summarises the capabilities of each generic modelling technique. The two sets of experiments revealed capabilities and characteristics of the modelling techniques. For example, the type of manufacturing system developments that could easily be modelled by a technique, along with relationships of model build-time and accuracy. These relationships are illustrated in Fig. 1. The capabilities are presented in detail in [8], and summarised as follows.

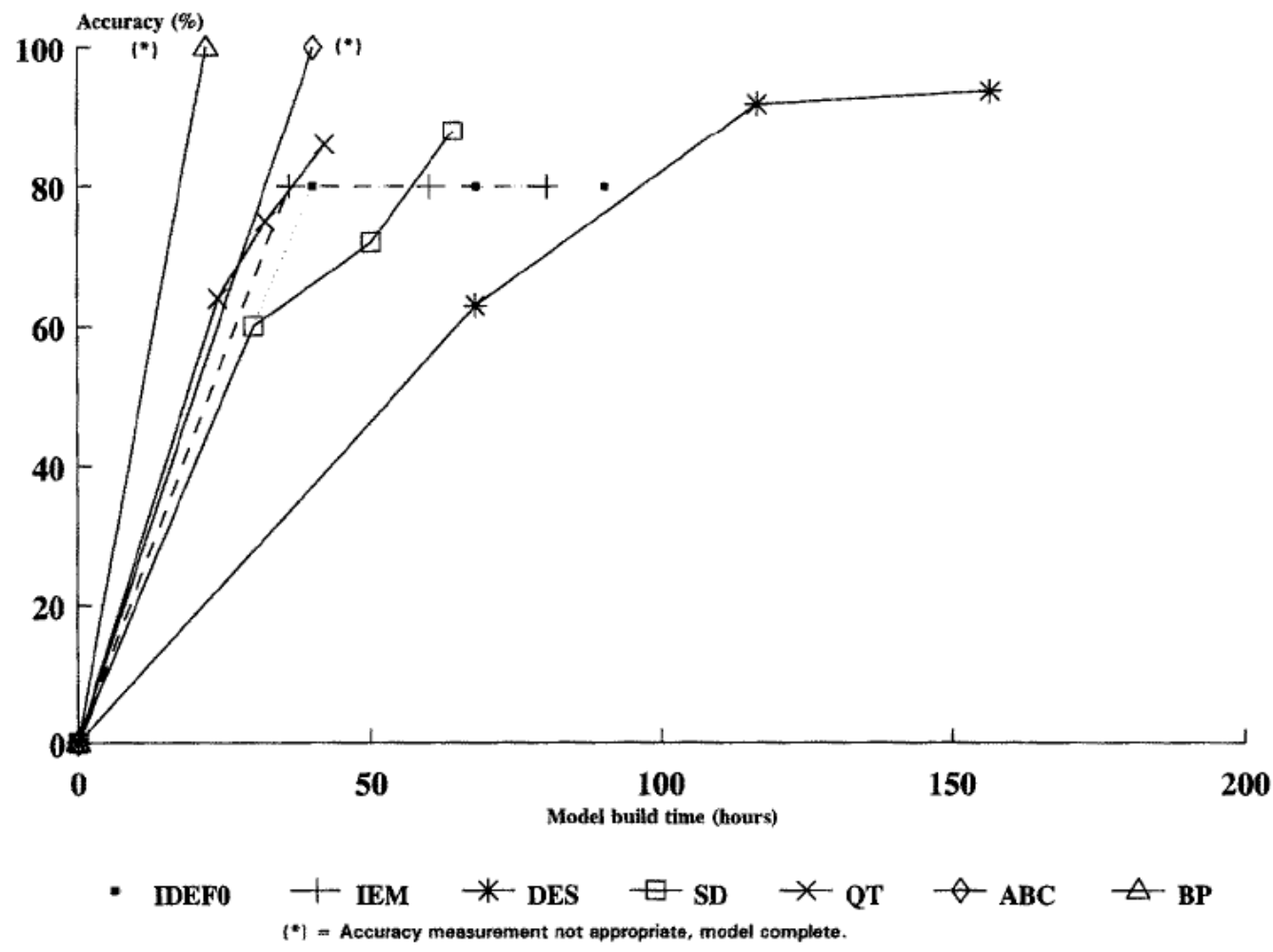

Fig. 1. Model build time and accuracy relationships 


\subsection{IDEFo}

The IDEF $F_{0}$ technique supports the construction of a form of flowchart that illustrates the activities resident within a system, see for example Brovoco and Yadav [10]. This representation is characterised through a specific diagrammatic syntax and a hierarchical structure (decomposition) for illustrating varying levels of detail in a systems description. Figure 2 shows one level of a model built in this study.

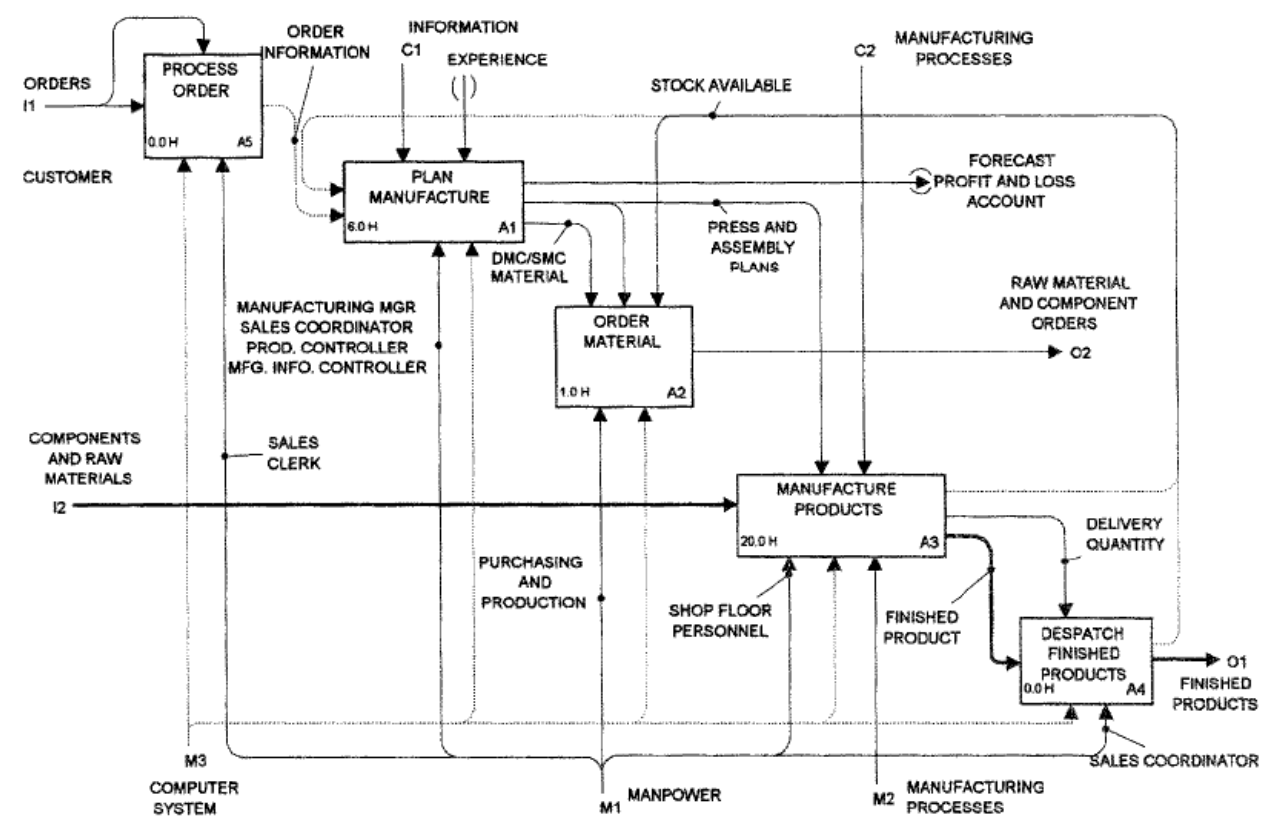

Fig. 2. A portion of the IDEFo model of the collaborating company.

The tool chosen to apply IDEF outomates the drawing of an IDEF functionality. This functionality allows numerical attributes to be assigned to each representation of a system activity. These attributes are then aggregated to one value at the highest level of the hierarchical structure. For example, the time for a shop floor assembly activity may be specified, then this value, along with time for other activities, may be aggregated to an overall value for product lead time. Although, strictly, IDEFo contains no mathematical features, these have been considered as they are becoming increasingly popular in modelling tools and appear to have significant potential. However, the principal limitations that were revealed through experimentation were mainly concerned with this mathematical functionality.

If the mathematical functionality is ignored then it can be concluded that IDEFO provides a strong mechanism for illustrating the activities that exist within a manufacturing system and how these activities interact at an instance in time. The diagrammatic syntax does cause some issues with model build time, model validation and credibility, mainly because to "read" a model the "reader" has to be conversant in this syntax. Conversely, this syntax does ensure rigorous model construction.

Unfortunately, the mathematical functionality is only superficial. Although it is possible to capture data about a system's performance at an instance in time, because only simple assumptions are made about the interactions of activities, the user must be very cautious of the apparent change in model performance if the model is modified. The main reason for this is that the control arrows on the diagrams have no effect on the structure of the calculation taking place within the model. This is demonstrated in lead-time calculations where product throughput is shown within the model to be constrained by machine-tool availability, but product queuing that should have occurred did not. tn practice, the mathematical capability is only useful as a mechanism to capture and refine, through decomposition, the model builder's anticipation of a system's performance.

In conclusion, IDEFO provides a useful mechanism for illustrating the static content and structure of a system. Furthermore, the numerical capabilities that are becoming common in modelling tools are helpful in data collection. 
This is, however, quite different from, and should not be confused with, the predictive functionality associated with modelling techniques such as DES, QT and SD.

\subsection{Enterprise Modelling (EM)}

IDEFO is a typical example of a classical structured systems analysis and design technique (SADT). Recently, a number of SADT type innovations have focused on the activity of business process re-engineering, see for example Johansson et al [11]. A variety of techniques have been designed to bridge the features of IDEFo and less abstract techniques such as material flowcharts, and these can be collectively termed enterprise modelling techniques.

The significant differences in performance between IDEFo and EM techniques are concerned with model credibility and build time. The less abstract nature of EM, coupled with a syntax that was found to be simpler to understand, meant that models were better accepted at the collaborating company. Hence, model credibility was recorded as being higher. The lower syntax constraints also meant that model building was faster. However, this relaxation in syntax did mean that there was less distinction between, for example, information controls on an activity, and information processed by an activity. In this sense, IDEFo could be considered to provide a richer system description.

The results for EM and IDEFO are similar in many respects, with only model build time and credibility differing significantly. While other differences do exist, when taken in a broad context of models, as with this study, these differences are not significant.

\subsection{Discrete Event Simulation (DES)}

DES models attempt to emulate the time-dependent behaviour of a real system by acting through the activities that occur in the real system, see for example Law and Kelton [12]. Invariably, the number of activities will be rationalised to improve modelling efficiency. Likewise, the model execution time is reduced by approximating actual activities to discrete events, time is then scaled down, and skipped forward according to an event list.

The experimentation programme revealed that there were three significant strengths of this technique. First, the technique could be applied to evaluate a wide variety of changes to a system. Secondly, the technique could provide information on the dynamic performance of a system. Thirdly, by computer animation of manufacturing system operation, the models produced by this technique were considered to have good credibility.

The most severe limitation of DES was found to be the time taken to build a model. Model building is concerned with transposing the knowledge about a system into a form suitable for model construction. In the experimentation programme, the time taken in model building with the DES technique was found to be considerably longer than for other modelling techniques in a similar situation, as shown in Fig. 1. The reason for this length of time is thought to be that DES encourages the construction of a more lifelike replication of the real system than do other techniques. As a consequence, the technique can appear to be ${ }^{10}$ data hungry". Other techniques have in-built approximations that reduce model build time, but thereby compromise relative flexibility.

In conclusion, experimentation revealed three significant strengths of the DES modelling technique, and these are accuracy, flexibility and credibility. However, model build and execution times may be considerably longer than for other modelling techniques in a similar situation.

\subsection{System Dynamics (SD)}

$\mathrm{SD}$ is a modelling technique where the progression of materials or information through a system is considered as a continuous flow, see for example Wolstenholme [13]. A system is described in terms of "resources" which flow through a variety of "states" according to "rates". An example of a resource is a product family, various states could be work in progress stores, and rates could be a representation of machines. Once the content of a system is defined, a mathematical expression is constructed to link rates with states. This expression is then executed at various time intervals, and performance of the system is recorded. 
Experimentation showed the time taken to build a SD model to be roughly half that required for a comparable DES model, as shown in Fig. 1. However, it also became clear that SD flexibility, and the absolute level of accuracy, are less than DES. This limitation is caused by the approximation inherent to SD, that all systems can be represented by a continuous flow. The severity of this approximation is dependent on the detail to which a system is being modelled. The SD model in the study could not cope, at its lowest level, with the sequencing of products or product batch size. However, at a higher level of aggregation, complex issues such as material control rules could be evaluated. Hence, this does encourage the practitioner to view a system at the highest possible level of aggregation. This is felt to be conducive to strategy evaluation. In conclusion, SD is relatively flexible if the model builder can approximate the effect of a development into a constraint on product flow. The predominant concern is that model accuracy and flexibility is limited by the inherent approximation of product manufacture to flows.

\subsection{Queuing Theory (QT)}

This technique is a mathematical determination of performance, based on the manufacturing system characteristics and required product volume, see for example Suri and Diehl [5]. The technique consists of a number of mathematical expressions of a standard form. These expressions are then populated with information about the system being modelled. Execution of these equations then provides values on the predicted average performance of the real system. The modelling tool used in this study divorced the engineer ; from the mathematical functions by initiating the input of information in a user friendly manner. The principal advantage of this mathematical approach to modelling is the time taken for model construction and execution. Experiments showed that typically a QT model could be constructed in a third of the time taken for a DES model of comparable accuracy. Furthermore, the model execution time, under comparable conditions, was only $1 \%$ of the time taken by a DES model. During these comparisons, it was discovered that when limits of model accuracy were explored, contrary to what was expected from the relatively large DES model, on average the QT model exhibited an almost comparable performance.

There are, however, a number of limitations with the QT approach. For example, the performance measures are given for conditions of steady-state system behaviour. Hence, periods of system transition that occur in a manufacturing system, when, for example, a new process is introduced, can only be modelled as crude time phases. Another limitation is that the routeing of parts through a system is approximated by a percentage rule. This was particularly apparent in the industrial study where one, short lead-time, product was always given priority on manufacturing operations in the real system. The QT model could not be configured to represent this situation. Finally, the technique lacked credibility as no graphical description of the system could be provided.

tn conclusion, there are a number of limitations with the QT modelling technique. The predominant concerns are that performance measures are given for conditions of steady-state system behaviour, that the inherent approximations restrict the depth and breadth to which a manufacturing system can be modelled, and that credibility is weak because graphical animation cannot be provided. The advantage is that a reasonably accurate model can be constructed quickly.

\subsection{Business Planning (BP)}

Business planning techniques focus on giving information that describes the financial performance of an organisation, see for example Gray [14]. As a result, measures such as profit, cash flow, turnover, etc., are provided. The technique requires information about a manufacturing system's performance, e.g. lead times, product cost for materials, overheads and labour, to be input. Therefore, although the technique appeared sufficiently flexible to consider a wide variety of manufacturing scenarios relatively quickly, in many cases the modeller had to carry out considerable synthesis to provide input data to a sufficiently high level.

The BP technique dynamically considers the change in content of a manufacturing system. For example, a change in fixed assets at a specific date could be introduced. Furthermore, a sequence of changes could be considered along with associated funding. The sensitivity of these investments could then be established in terms of, for example, sales growth or increases in interest rates. 
In conclusion, the strength of BP is that it provides a business perspective of manufacturing developments. The predominant weaknesses are that valid flexibility and credibility are limited because the capabilities of a manufacturing system are only superficially considered.

\subsection{Activity Based Costing (ABC)}

It may be expected that $A B$ is solely focused on establishing product cost, see for example Kaplan [15]. However, in practice, ABe can also be used to examine the "overhead" cost structure in an organisation. Furthermore, ABe can either be used as an operational costing system or for taking a snapshot to provide relatively accurate information for strategic decision making. The latter approach has been taken in this study.

The constraining issue in the application of ABe is the time taken to build a valid model. In this study, the model build time was found to consist principally of two activities, data interpretation from the company accounts, and choosing appropriate cost drivers. The management accounts at the collaborating company contained cost centres that were mainly convenient groupings of costs and not necessarily cost pools in the activity based costing sense. Choosing the appropriate cost drivers also took considerable time. The activity was simplified by attempting only to apportion larger costs accurately and by keeping the number of cost drivers down to a reasonably small number. Using this strict approach, once data had been collected, a coarse $A B C$ model could be constructed the collaborating company in 40 working hours.

In conclusion, $\mathrm{ABC}$ is focused on providing product cost, and has the flexibility to assess a range of strategic developments in terms of this measure. Contrary to some evidence in the literature, this modelling technique can be applied in a reasonably short time if restricted to a strategic level.

\section{Conclusion}

We have considered how popular but diverse modelling techniques support manufacturing strategy evaluation. The findings are based on experimental work, and can be summarised as follows.

$\mathrm{IDEF}_{\mathrm{o}}$ and enterprise modelling are strong mechanisms for illustrating the activities in a system, and their interactions, at an instant in time. Experimentation revealed limitations with the mathematical, and hence predictive, capabilities of these modelling approaches.

Discrete event simulation can evaluate a wide variety of issues, to a low level of detail, with relatively high model accuracy, and good model credibility. System dynamics has slightly less flexibility but it exhibits a relatively rapid model build rate and model execution tine. However, because of the inherent approximation of treating a product as a flow, the model detail, credibility, and absolute level of accuracy are less than for DES. Queuing theory enables an accurate model to be constructed and executed in a fraction of the time taken for comparable DES and SD models. The predominant concerns are that performance measures are given for conditions of steady-state system behaviour, and that the inherent approximations restrict flexibility.

Activity based costing and business planning are both financial modelling techniques. $A B C$ is focused on providing product cost, and has the flexibility to assess a range of strategic developments. Models can be built relatively quickly if they are restricted to a strategic snapshot of manufacturing system performance. BP provides a broad financial perspective of manufacturing developments, but flexibility and credibility are limited because the manufacturing system characteristics are only superficially considered.

The strength of this review emanates from its rigorous experimental nature, rather than being based on the unsupported assertions so often voiced and found in the literature. This is a strength that should be built upon. Further empirical work is needed to provide a broad and robust body of knowledge about modelling to which practising engineers can confidently refer. 


\section{References}

1. T. S, Baines, D. J. Hamblin and D. K. Harrison, "A unified classification of manufacturing strategies and design processes", IEE Engineering Management Journal, 3(6), pp. 281-286, 1993.

2. K. W. Platts, "Manufacturing audit in the process of strategy formulation", PhD Thesis, University of Cambridge, 1990.

3. C. W. Hofer and D. Schendel, Strategy Formulation: Analytical Concepts, West, 1978.

4. H. Mintzberg, D. Raisinghani and A. Theoret, "The structure of unstructured decision processes", Administrative Science Quarterly, 21, pp. 246-275, June 1976.

5. R. Suri and G. W. Diehl, "'A precursor to simulation for complex manufacturing systems." in D. Gantz, G. Blais and S. Solomon (ed.), Proceedings of the 1985 Winter Simulation Conference, pp. 4t 1-420, 1985.

6. A. Cm-rie, Simulation of Manufacturing Systems, Wiley, 1988. 7. S. Cooke and N. Slack, Making Management Decisions. Prentice Hall. 191.

7. T. S. Baines, "Modelling in the evaluation of a manufacturing strategy," PhD thesis, Cranfield University, 1995.

8. T. S. Baines, J. M. Kay, D. J. Hamblin and D. K. Harrison, "A taxonomy of modelling techniques for the analysis of a manufacturing strategy, The MATADOR Conference, UMIST, pp. 29-34, April 1995.

9. R. R. Brovoco and S. B. Yadav, "A methodology to model the functional structure of an organisation", Computers in Industry, 6, pp. 345361, October 1985.

10. H. J. Johansson, P. McHugh, A. J. Pendlebury and W. A. Wheeler, Business Process Reengineering, John Wiley, 1993.

11. A. M. Law and W. D. Kelton, Simulation Modeling and Analysis, McGraw-Hill, 1991.

12. E. F. Wolstenholme, System Enquiry: A System Dynamics Approach, John Wiley, 1990.

13. P. Gray, "Using the interactive financial planning", pp. 286-292, December 1984.

14. R. S. Kaplan, "Yesterday's accounting undermines production", Harvard Business Review, pp. 95-101, July/August 1984. 